\title{
A Study and Review on Fingerprint Image Enhancement and Minutiae Extraction
}

\author{
Atul S. Chaudhari ${ }^{1}$, Sandip S. Patil ${ }^{2}$ \\ ${ }^{\text {I }(D e p a r t m e n t ~ o f ~ C o m p u t e r ~ E n g i n e e r i n g, ~ S S B T ' s ~ C o l l e g e ~ o f ~ E n g i n e e r i n g ~ \& ~ T e c h n o l o g y, ~ B a m b h o r i, ~ J a l g a o n ~}$ \\ [M.S.], India) \\ ${ }^{2}$ (Department of Computer Engineering, SSBT's College of Engineering \& Technology, Bambhori, Jalgaon \\ [M.S.], India)
}

\begin{abstract}
Fingerprint recognition is one of the most popular and successful methods used for person identification, which takes advantage of the fact that the fingerprint has some unique characteristics called minutiae; which are points where a curve track finishes, intersect with other track or branches off. However obtaining a good fingerprint image is not always easy. Fingerprint images are rarely of perfect quality. They may be degraded and corrupted due to variations in skin and impression conditions. Thus, image enhancement techniques are employed prior to minutiae extraction to obtain a more reliable estimation of minutiae locations. In this paper we are doing the study and analysing the performance of various fingerprint enhancement techniques.
\end{abstract}

Keywords: Fingerprint, Minutiae, Fingerprint enhancement, Binarisation, Thinning, Minutiae Extraction.

\section{Introduction}

The word biometrics is derived from the Greek words bios (meaning life) and metron (meaning measurement); biometric identifiers are measurements from living human body. [3] Perhaps all biometric identifiers are a combination of anatomical and behavioral characteristics and they should not be exclusively classified into either anatomical or behavioral characteristics. For example, fingerprints are anatomical in nature but the usage of the input device depends on the person's behavior. Thus, the input to the recognition engine is a combination of anatomical and behavioral characteristics.

Fingerprints are the patterns formed on the epidermis of the fingertip. Fingerprints are made up of series of ridges and valleys (also called as furrows) on the surface of the fingertip and have core around which pattern like swirls, loops or arches are curved to ensure that each print is unique. [3]

The fingerprint is composed of ridges and valleys. The interleaved pattern of ridges and valleys are the most evident structural characteristic of a fingerprint. The ridges are the single curved segment and valleys are the region between two ridges. The most commonly used fingerprint features are minutiae. Minutiae are the discontinuities in local ridge structure. They are used by forensic experts to match two fingerprints. There are about 150 different types of minutiae. [7] Among these minutiae types "ridge ending" and "ridge bifurcation" are the most commonly used as all the other types of minutiae are combinations of ridge endings and ridge bifurcations. A ridge ending is defined as the ridge point where a ridge ends abruptly. [3] A ridge bifurcation is defined as the ridge point where a ridge forks or diverges into branch ridges. [3] Some common types of minutiae are shown in fig 1.

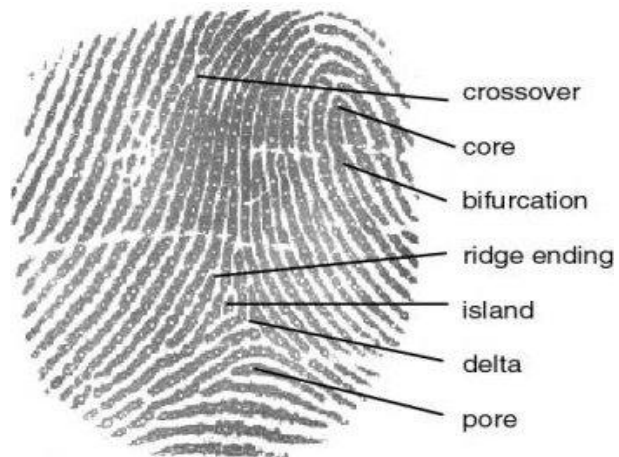

Fig 1: Types of minutiae.

II. Related Work

Lili Liu and Tianjie [1] Cao proposed an efficient verification system based on biometrics. In this system they have used Gabor filter based Enhancement and CN concept for Minutiae Extraction. 
Lin Hong, Anil Jain, Sharath PanKanti and R. Bolle[10] have designed and implemented an Identity Authentication system which operates in two stages: minutiae extraction and minutiae matching. An alignmentbased elastic matching algorithm is proposed for minutiae matching.

Manvjeet Kaur, Mukvinder Singh and Parvinder S. Sindhu [9] proposed Fingerprint Verification System using Minutiae Extraction Technique. In this System they have introduced combined methods to build a minutia extractor and a minutia matcher. Segmentation with Morphological operations used to improve thinning, false minutiae removal, minutia marking. For this system they have used Histogram Equalization and FFT for fingerprint image enhancement and $\mathrm{CN}$ Concept for Minutiae Extraction.

F. A. Afsar, M. Arif and M. Hussain [5] presented the minutiae based Automatic Fingerprint Identification Systems. The technique is based on the extraction of minutiae from the thinned, binarized and segmented version of a fingerprint image. The system uses fingerprint classification for indexing during fingerprint matching which greatly enhances the performance of the matching algorithm.

Ishpreet Singh Virk and Raman Maini [11] have used histogram equalization for fingerprint image enhancement, segmentation using Morphological operations, minutia marking by specially considering the triple branch counting, branch into three terminations, an alignment-based elastic matching algorithm minutia unification by decomposing has been developed for minutia matching were implemented. The proposed alignment- based elastic matching algorithm is capable of finding the correspondences between minutiae without resorting to exhaustive search.

Various fingerprint enhancement and matching algorithm have been proposed in literature. Most of the algorithm has no difficulty in matching good quality fingerprint but matching low quality fingerprint remain a challenging problem. Table 1 shows review of various fingerprint enhancement and matching techniques.

Table 1: Review of Various Fingerprint Enhancement and Minutiae Matching Techniques

\begin{tabular}{|c|c|c|c|c|c|}
\hline Authors & Techniques Used & $\begin{array}{l}\text { FAR, FRR } \\
\text { and VR }\end{array}$ & D/B Used & $\begin{array}{l}\text { Accurac } \\
\mathbf{y}\end{array}$ & $\begin{array}{l}\text { Comput } \\
\text { ation } \\
\text { Time }\end{array}$ \\
\hline $\begin{array}{l}\text { Lili Liu and } \\
\text { Tianjie Cao } \\
(2012)[1]\end{array}$ & $\begin{array}{l}\text { Gabor filter based } \\
\text { Enhancement and CN } \\
\text { concept for Minutiae } \\
\text { Extraction }\end{array}$ & $\begin{array}{l}\text { FAR }=0.085 \\
\% \\
\text { FRR }=1.4 \% \\
\text { VR }=99.75 \%\end{array}$ & $\begin{array}{l}2000 \text { fingerprint } \\
\text { images of } 200 \\
\text { individuals at } \\
500 \mathrm{dpi} \\
\text { size: } 256 \times 360\end{array}$ & N/A & N/A \\
\hline $\begin{array}{l}\text { Lin Hong, } \\
\text { Anil Jain, } \\
\text { Sharath } \\
\text { PanKanti and } \\
\text { R. Bolle } \\
\text { (1997) [10] }\end{array}$ & $\begin{array}{l}\text { Alignment based elastic } \\
\text { matching algorithm }\end{array}$ & $\mathrm{FRR}=15 \%$ & $\begin{array}{l}\text { MSU: contain } 10 \\
\text { images per finger } \\
\text { from } 70 \\
\text { individuals. Total } \\
700 \text { images } \\
\text { size: } 640 \times 480\end{array}$ & High & $\begin{array}{l}1.4 \text { sec. } \\
\text { on Sun } \\
\text { ULTRA } \\
1 \text { Work } \\
\text { station }\end{array}$ \\
\hline $\begin{array}{l}\text { Manvjeet } \\
\text { Kaur, } \\
\text { Mukvinder } \\
\text { Singh and } \\
\text { Parvinder S. } \\
\text { Sindhu } \\
\text { (2008) [9] }\end{array}$ & $\begin{array}{l}\text { Histogram } \\
\text { Equalization and FFT for } \\
\text { enhancement and CN } \\
\text { Concept for Minutiae } \\
\text { Extraction }\end{array}$ & $\mathrm{VR}=75 \%$ & N/A & N/A & N/A \\
\hline $\begin{array}{l}\text { F. A. Afsar, } \\
\text { M. Arif and } \\
\text { M. Hussain } \\
\text { (2004) [5] }\end{array}$ & $\begin{array}{l}\text { Gabor filter based } \\
\text { Enhancement and CN } \\
\text { concept for Minutiae } \\
\text { Extraction }\end{array}$ & $\begin{array}{l}\mathrm{FAR}=1 \% \\
\mathrm{FRR}=7 \%\end{array}$ & $\begin{array}{l}\text { FVC } 2000 \\
800 \text { fingerprints } \\
\text { from } 110 \text { different } \\
\text { fingers }\end{array}$ & High & $12 \mathrm{sec}$. \\
\hline $\begin{array}{l}\text { Ishpreet Singh } \\
\text { Virk and } \\
\text { Raman Maini } \\
(2012)[11]\end{array}$ & $\begin{array}{l}\text { Histogram } \\
\text { Equalization for } \\
\text { enhancement and } \mathrm{CN} \\
\text { Concept for Minutiae } \\
\text { Extraction }\end{array}$ & $\begin{array}{l}\mathrm{FAR}=0.06 \% \\
\mathrm{FRR}=6.9 \%\end{array}$ & FVC2000 & N/A & N/A \\
\hline
\end{tabular}

\section{Fingerprint Image Preprocessing}

Fingerprint Image preprocessing consist of following steps:

1. Segmentation

2. Fingerprint Image Enhancement

3. Binarisation 


\section{Segmaentation}

Segmentation is the process of separating the foreground regions in the image from the background regions. The foreground regions correspond to the clear fingerprint area containing the ridges and valleys, which is the area of interest. The background corresponds to the regions outside the borders of the fingerprint area, which do not contain any valid fingerprint information. [8] When minutiae extraction algorithms are applied to the background regions of an image, it results in the extraction of noisy and false minutiae. Thus, segmentation is employed to discard these background regions, which facilitates the reliable extraction of minutiae. In a fingerprint image, the background regions generally exhibit a very low grey-scale variance value, whereas the foreground regions have a very high variance. Hence, a method based on variance thresholding can be used to perform the segmentation. Firstly, the image is divided into blocks and the grey-scale variance is calculated for each block in the image. If the variance is less than the global threshold, then the block is assigned to be a background region; otherwise, it is assigned to be part of the foreground. The grey-level variance for a block of size $\mathrm{W} \times \mathrm{W}$ is defined as:

$$
V(k)=\frac{1}{W^{2}} \sum_{i=0}^{W-1} \quad \sum_{j=0}^{W-1}(I(i, j)-M(k))^{2} \quad \text { Eq: (1) }
$$

where $\mathrm{V}(\mathrm{k})$ is the variance for block $\mathrm{k}, \mathrm{I}(\mathrm{i}, \mathrm{j})$ is the grey-level value at pixel $(\mathrm{i}, \mathrm{j})$, and $\mathrm{M}(\mathrm{k})$ is the mean greylevel value for the block $\mathrm{k}$.

\section{Fingerprint Image Enhancement}

Fingerprint Enhancement is used to improve the image quality by removing noise by low-pass filter. The configuration of parallel ridges and furrows with well-defined frequency and orientation in a fingerprint image provide useful information which helps in removing undesired noise. The sinusoidal-shaped waves of ridges and furrows vary slowly in a local constant orientation. The band pass filter i.e. tuned to the corresponding frequency and orientation can effectively remove the undesired noise and preserve the true ridge and furrow structures. An image is then passed through the filtering stage. Gabor filters have both frequencyselective and orientation-selective properties and have optimal joint resolution in both spatial and frequency domains. Therefore, it is appropriate to use Gabor filters as band pass filters to remove the noise and preserve true ridge/valley structures. [4]

\section{Binarisation}

Most minutiae extraction algorithms operate on binary images where there are only two levels of interest: the black pixels that represent ridges, and the white pixels that represent valleys. Binarisation is the process that converts a grey level image into a binary image. This improves the contrast between the ridges and valleys in a fingerprint image, and consequently facilitates the extraction of minutiae.

Usually grayscale image is converted into binary image using a global threshold. [4] The binarisation process involves examining the grey-level value of each pixel in the enhanced image, and, if the value is greater than the global threshold, then the pixel value is set to a binary value one; otherwise, it is set to zero. The outcome is a binary image containing two levels of information, the foreground ridges and the background valleys.

Let $\mathrm{I}(\mathrm{x}, \mathrm{y})$ represent the intensity value of enhanced grayscale image at pixel position $(\mathrm{x}, \mathrm{y})$. Let Tp be the threshold value. In case of fingerprint images $\mathrm{Tp}$ represents the differentiating intensity between the background pixels and ridge pixels. $\mathrm{BW}(\mathrm{x}, \mathrm{y})$ represent the binary image obtained by the equation.

$$
B W(x, y)=\left\{\begin{array}{lr}
1, & \text { if } I(x, y) \geq T_{p} \\
0, & \text { Otherwise }
\end{array} \quad\right. \text { Eq: (2) }
$$

\section{Minutiae Extraction}

The minutiae extraction steps are explained below:

\section{Thinning}

Thinning is a morphological operation that successively erodes away the foreground pixels until they are one pixel wide. [2] A standard thinning algorithm is employed, which performs the thinning operation using two sub-iterations. This algorithm is accessible in MATLAB via the 'thin' operation under the bwmorph function. [2] Each sub-iteration begins by examining the neighbourhood of each pixel in the binary image, and based on a particular set of pixel-deletion criteria, it checks whether the pixel can be deleted or not. These subiterations continue until no more pixels can be deleted. The application of the thinning algorithm to a fingerprint image preserves the connectivity of the ridge structures while forming a skeletonised version of the binary image. This skeleton image is then used in the subsequent extraction of minutiae. 


\section{Minutiae Detection}

The most commonly employed method of minutiae extraction is the Crossing Number (CN) concept. This method involves the use of the skeleton image where the ridge flow pattern is eight-connected. The minutiae are extracted by scanning the local neighborhood of each ridge pixel in the image using a $3 \times 3$ window. The $\mathrm{CN}$ value is then computed, which is defined as half the sum of the differences between pairs of adjacent pixels in the eight-neighborhood. According to Rutovitz the $\mathrm{CN}$ for a ridge pixel $\mathrm{P}$ is given by: [6][11]

$$
C N=\frac{1}{2} \sum_{i=1}^{8}\left|P_{i}-P_{i-1}\right|, \quad P_{9}=P_{1} \quad \text { Eq: (3) }
$$

where $\mathrm{Pi}$ is the pixel value in the neighborhood of $\mathrm{P}$. For a pixel $\mathrm{P}$, its eight neighbouring pixels are scanned in an anti-clockwise direction as follows:

\begin{tabular}{|l|l|l|}
\hline $\mathrm{P}_{4}$ & $\mathrm{P}_{3}$ & $\mathrm{P}_{2}$ \\
\hline $\mathrm{P}_{5}$ & $\mathrm{P}$ & $\mathrm{P}_{1}$ \\
\hline $\mathrm{P}_{6}$ & $\mathrm{P}_{7}$ & $\mathrm{P}_{8}$ \\
\hline
\end{tabular}

Fig 3: $3 \times 3$ window for searching minutiae [6] [11]

After the $\mathrm{CN}$ for a ridge pixel has been computed, the pixel can then be classified according to the property of its $\mathrm{CN}$ value. Using the properties of the $\mathrm{CN}$ as shown in Table 2, the ridge pixel can then be classified as a ridge ending, bifurcation or non-minutiae point. For example, a ridge pixel with a $\mathrm{CN}$ of one corresponds to a ridge ending, and a $\mathrm{CN}$ of three corresponds to a bifurcation.

Table: 2 Properties of Crossing Number [6] [11]

\begin{tabular}{|c|l|}
\hline $\mathbf{C N}$ & \multicolumn{1}{|c|}{ Property } \\
\hline 0 & Isolated Point \\
\hline 1 & Ridge Ending Point \\
\hline 2 & Continuing Ridge Point \\
\hline 3 & Bifurcation Point \\
\hline 4 & Crossing Point \\
\hline
\end{tabular}

\section{Conclusion}

The performance of a fingerprint feature extraction and matching algorithms heavily depends upon the quality of the input fingerprint image. Various enhancement approaches such as Histogram equalization, FFT, have shown to improve the fingerprint image quality and recognition performance in different studies. Gabor filters have both frequency-selective and orientation-selective properties. It is observed that Gabor filter method of fingerprint image enhancement is giving better results. Minutiae extraction algorithm can detect all the minutiae, including both true and false minutiae, using the Rutovitz Crossing Number $(\mathrm{CN})$ on the skeleton images after thinning stage.

\section{References}

[1] Lili Liu and Tianjie Cao, "The Research and Design of an Efficient Verification System Based on Biometrics", International Conference on Computer Science and Electrical Engineering, 2012.

[2] Dr. Salah M. and Dr. Feryal I. Haj Hassan, "Fingerprint Minutiae Extraction”, Journal of Computing Press., Vol. 2, November 2010.

[3] Maltoni D., Maio D., Jain A. K., and Prabhakar S. "Handbook of Fingerprint Recognition”. Springer, 2003.

[4] Lavanya B. N., K. B. Raja and Venugopal K. R., "Minutiae Extraction in Fingerprint using Gabor Filter Enhancement", International Conference on Advances in Computing, Control and Telecommunication Technologies, IEEE, 2009.

[5] F. A. Afsar, M. Arif and M. Hussain, "Fingerprint Identification and Verification System using Minutiae Matching", National Conference on Engineering Technologies, 2004.

[6] Feng Zhao and Xiaoou Tang, "Preprocessing and postprocessing for skeleton-based fingerprint minutiae extraction", Pattern Recognition Society, Published by Elsevier Ltd, 2006

[7] L. Hong, Y. Wan and A. Jain, "Fingerprint Image Enhancement: Algorithm and Performance Evaluation", IEEE Pattern Analysis and Machine Intelligence, vol. 20, No. 8, pp. 777-787, 1998.

[8] Raymond Thai," Fingerprint Image Enhancement and Minutiae Extraction" technical Report, The University of Western Australia, 2003.

[9] Manvjeet Kaur, Mukvinder Singh and Parvinder S. Sindhu, "Fingerprint Verification System using Minutiae Extraction Technique", Proceedings of World Academy of Science, Engineering and Technology, Vol. 36, December 2008.

[10] Lin Hong, Anil Jain, Sharath PanKanti and R. Bolle, "Identity Authentication Using Fingerprints", Proceedings of the IEEE, Vol. 85, September1997.

[11] Ishpreet Singh Virk and Raman Maini, "Fingerprint Image Enhancement and Minutiae Matching in Fingerprint Verification", Journal of Computing Technologies, vol. 1, June 2012. 